\section{CHEMISTRY RESEARCH BOARD}

A

REPORT of the Chemistry Research Board which prefaces the report of the Director of the Chemical Research Laboratory for the year 1946 covers the nine-year period January 1,1938 , to December 31, 1946*, and thus presents a summary of the more important developments since the publication of the second report of the Board, which covered the three years ending December 31, 1937. A fuller account is promised in a further report which is still in preparation.

Both the Board's report and that of the Director devote considerable space to discussion of the postwar plans for the work of the Laboratory. The Chemistry Research Board proposed that the work of the Laboratory should be concentrated on a few well-selected topics in the fields of coal tar, corrosion of metals, inorganic chemistry, high polymers and plastics, organic intermediates and microbiology. For the present, owing to limitations of space, it is proposed that microbiological studies should be concentrated on corrosion problems, and the Board recommends that throughout its work the Laboratory should pay special attention to the development of new techniques. To implement this programme, expenditure at the rate of $£ 70,000$ per annum will be required as well as non-recurrent expenditure on

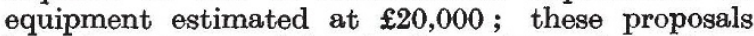
have been approved by the Committee of the Privy Council for Scientific and Industrial Research.

The Director of the Laboratory in his report details the objectives of the work of the five main research sections in which the scientific staff of twenty-five scientific officers, thirty-three experimental officers and assistants, and twenty-five laboratory assistants has been organised to implement these proposals. There are also two nonsectional groups for exploratory and special work under the Director and Assistant Director, respectively. The present objectives comprise the investigation of corrosion in immersed and atmospheric conditions with the view of the prevention of rusting, tarnishing and corrosion of ferrous and non-ferrous metals in industrial use; the investigation of the microbiological corrosion of metals, particularly in soil, by the influence of bacteria which reduce sulphates, and the investigation of ad hoc corrosion problems of industrial importance. In the inorganic field they include the investigation of semi-rare metals, especially their isolation from wastes and low-grade sources, the study and development of physical methods of analysis, and development of micro-analytical methods and other new techniques; in that of high polymers and plastics, the determination of the relations between structure and properties and the preparation of improved plastic materials such as glues and ion-exchange resins; and in the coal tar field, the isolation and identification of tar constituents using the newest methods of separation and analysis, determination of the physical and physico-chemical constants of tar constituents and derived or related substances, and the development of new uses for tar products and their derivatives. The programme also includes the study of high-pressure operations and the production of useful chemicals by such means, and the preparation of compounds capable of polymerization

* Department of Scientiflc and Industrial Research. Report of the Chemistry Research Board for the Period 1938-1946, with Report of the Director of the Chemical Research Laboratory for the Year 1946 . Pp. iv +68 . (London: H.M. Stationery Office, 1948.) 18. 3d. net. or co-polymerization, particularly those containing silicon.

The Board's report, as well as that of the Director, refers to some of the directions in which progress has been made on these main items during 1946, and the latter report describes the new work done by the non-sectional groups in collaboration with the Agricultural Research Council on the formulation of salves or smears for control of ticks and in devising a new micro method fcr the rapid determination of D.D.T. Satisfactory results have also been obtained by this section in work on blow fly control. In the Engineering Services, the Corrosion Section has redesigned the coupling used for the slow rotation of specimens so as to ensure positive and concentric alignment of the spindles to the shafts, and a sectional view of the arrangement is included in the report. Investigations of the corrosion-rate of mild steel in salt solution using the high-speed rotor suggest that the nature of the surface finish may appreciably influence corrosion-rates and the reproducibility of results.

The Corrosion of Metals Section has continued to develop both the high-speed and the low-speed rotor techniques for immersed conditions, and with the former technique has investigated the influence of surface preparation on the reproducibility of corrosion-xate and the influence of movement on the corrosion-rate of mild steel in fresh water. Using the new endless belt and beaker type of apparatus for accelerated atmospheric corrosion tests recently noted (Nature, 162,$90 ; 1948$ ) the corrosion of steel in salt water atmospheres and the effect of impact damage on the corrosion of painted steel have been examined. In collaboration with the Light Division of the National Physical Laboratory, a light-reflexion meter, utilizing the integrating sphere principle, has been constructed, and in collaboration with the British Iron and Steel Recearch Association the corrosion behaviour of a comprehensive series of low alloy steels is being investigated, using the oxygenabsorption apparatus and the low-speed rotor apparatus. An extensive search is being made for organic corrosion inhibitors more efficient than sodium benzoate. Other work has been concerned with wrapping materials and with vapour-phase inhibitors, as well as with the influence various methods of surface treatment have on the corrosion resistance of mild steel and on the protection afforded by applied coatings.

In the Inorganic Section an attempt is being made to correlate the rare metal content of flue dust and coal ash with that of the fuel from which the dust or ash originated; and methods of extraction of germanium and gallium from flue dust are being studied. The physical chemistry group has given attention to detection and possible estimation of some minor metals by fluorescent technique; and in work on foams for fire fighting, carried out for the Ministry of Home Security, a portable foam meter has been designed which has been very useful in large-scale experiments with petrol fires.

The High Polymers and Plastics Section's comprehensive study of the mechanical properties of reinforced plastics, commenced, jointly with the Engineering Division of the National Physical Laboratory, during the War, has led to a clearer understanding of the relationships existing between the mechanical properties of laminated plastics and the properties of their components. In connexion with the study of weathering properties, a more convenient 
apparatus is being constructed for the accurate determination of the sorption isotherms of phenolformaldehyde resins, and the study of the relation between structure and dielectric properties of high folymers is being resumed. A critical review has been made of the literature dealing with the ionexchange properties of high polymers, and exploratory work commenced on the 'Wofatits'. In the field of adhesives, work has been carried out to improve the setting of resins from monohydric phenols and also of rescorcinol-formaldehyde resins. A cold-setting resorcinol-formaldehyde glue S.R.38, developed in 1944-45, has shown excellent strength and durability. A study has also been made of glues for light alloys.

The Coal Tar Section has completed and brought into operation an all-metal semi-scale fractionating column and has used the column in the preparation of a tar-oil fraction containing the two methylnaphthalenes. A high-vacuum pumping system has been set up to manipulate highly purified and hygroscopic materials. A new expression has been deduced for the relationship between differential changes of composition with boiling-point and applied to the quantitative calculations of the changes in composition with temperature of the azeotrope formed between ethyl acetate and ethyl alcohol. Results obtained in the separation of $2: 6$-butidine, $\beta$-picoline and $\gamma$-picoline by azeotropic distillation with acetic acid are recapitulated in the report, and a method worked out for the separation of crude xylole fractions has been applied to the examination of a number of crudes of different origin. Some work has been done on the fractionation of lutidine bases, and a method of estimating acenaphthylene in acenaphthene based on ultra-violet absorption spectrography has been applied to the determination of the acenaphthylene content of the crude material formed in the dehydrogenation of acenaphthene.

In the Organic Intermediates Section, preliminary work on organic compounds from boron was not promising; the chief attention was therefore given to the organic compounds from silicon, including the direct synthesis of methylchlorosilanes and their separation by fractional distillation and alcoholysis, and the preparation of organo-silica resins and elastomers. Results obtained in the hydrogenation of metallic salts of fatty acids indicate that lead salts may prove superior to those of copper.

The report of the Chemistry Research Board itself refers to many of these investigations, which are described in somewhat greater detail in the Director's report. The former report also mentions the establishment, on the Board's recommendation, of a joint panel on acetylene chemistry, with the Director of the Laboratory as chairman, between the Department of Scientific and Industrial Research and the Ministry of Supply, to consider the present position of the research on and development and production of acetylene in the United Kingdom and its utilization as an intermediate product in chemical industry. The Board has also recommended the resumption of work on the 'Oxo' reaction between olefins, carbon monoxide and hydrogen to yield aldehydes. Reference to the work of the Laboratory during the war years is very brief; but among its contributions to the war effort thus singled out for mention is the rotor test for assessing the relative value of a variety of protective coatings, the evaluation of anti-freeze materials and the discovery of the inhibitive properties of sodium benzoate, both as an addition to aqueous solutions and when incorporated in wrapping materials. A long series of experiments in the Laboratory showed that suitable carbon blacks could be prepared from indigenous material, particularly coal tar products, and a highly successful foam for fighting petrol fires was also developed from indigenous sources. Much attention was given to the development of a food yeast from Torula utilis as an insurance against possible shortage of protein food, and on the basis of this war-time research and development, the Colonial Office has now erected a full-scale plant in Jamaica. The shortage of derris led the Laboratory to collaborate with the Agricul. tural Research Council in the development of products for the control of animal parasites, and a satisfactory D.D.T. emulsion was finally formulated for the control of blow-fly on sheep.

Lists of papers published from the Laboratory during 1946 and of British patents and patent applications granted or filed during the year are appended to the report.

A summary account of current work at the Laboratory was published in Nature of July 17, p. 90.

\section{PRODUCTION OF INDOLE BY ESCHERICHIA COLI}

\section{By Dr. EDWIN A. DAWES}

\section{Department of Biochemistry, University of Leeds}

\section{$T$}

HE mechanism of the production of indole by

Escherichia coli has attracted the attention of many workers since Hopkins and $\mathrm{Cole}^{1}$ first demonstrated that the precursor of indole is tryptophan. The problem is an intriguing one among the bacterial degradation of amino-acids, inasmuch as fission of a carbon-carbon bond occurs at a position in the molecule remote from the amino- and carboxylgroups, instead of the more usual carbon-nitrogen split. It became even more intriguing when an exhaustive survey of theoretically possible intermediates in the reaction failed to give rise to indole in the presence of the organism.

In 1922, Frieber ${ }^{2}$ described a series of growth experiments in which tryptophan in the growth medium was replaced by various indole derivatives, and he found that no indole was produced from 3 -indolecarboxylic acid, 2 : 3-indoledicarboxylic acid, skatole, 3-indolealdehyde, 3-indoleacetic acid, $\beta-3$ indolepyruvic acid and $\beta$-3-indole-ethylamine. In spite of the failure of 3 -indoleacetic acid to give rise to indole he postulated that the reaction occurred in two stages, namely: (1) formation of 3-indoleacetic acid; and (2) the breakdown of this compound to indole; only the former occurring in the presence of fermentable carbohydrate.

$\mathrm{Harden}^{3}$, in 1930, gave the reaction as follows: Tryptophan $\rightarrow$ 3-3-indolepropionic acid $\rightarrow$ 3-indoleacetic acid $\rightarrow$ indole.

Extensive work on this subject appeared in 1935 by Woods ${ }^{4}$ who, using the washed viable cell suspension technique, demonstrated that five atoms of oxygen are required for the complete oxidation of one molecule of tryptophan, and that $D$-tryptophan is not metabolized; and he presented strong presumptive evidence for the anaerobic production of $\beta$-3-indolepropionic acid (cf. Hopkins and $\mathrm{Cole}^{5}$ ). He also investigated the action of cell suspensions of Esch. coli on various possible intermediates ${ }^{6}$, and he 